\title{
Building the foundation for culture change through the design, implementation, and assessment of an interprofessional education intervention
}

\author{
Christa Cerra ${ }^{* 1,2}$, Diana Drake ${ }^{3}$, Brian Sick ${ }^{4}$, Jean A. King ${ }^{5}$, Mary Chesney ${ }^{3}$, M. Nawal Lutfiyya ${ }^{6}$ \\ ${ }^{1}$ Renaissance Family Practice, University of Pittsburgh Medical Center, Pittsburgh, United States \\ ${ }^{2}$ Schools of the Health Sciences Health Policy Institute, University of Pittsburgh, Pittsburgh, United States \\ ${ }^{3}$ School of Nursing, University of Minnesota, Minneapolis, United States \\ ${ }^{4}$ Medical School, University of Minnesota, Minneapolis, United States \\ ${ }^{5}$ College of Education and Human Development, University of Minnesota, Minneapolis, United States \\ ${ }^{6}$ Academic Health Center, National Center for Interprofessional Practice and Education, University of Minnesota, Minneapolis, \\ United States
}

Received: August 21, 2014

DOI: $10.5430 /$ jnep.v5n1p46
Accepted: October 13, $2014 \quad$ Online Published: October 26, 2014

URL: http://dx.doi.org/10.5430/jnep.v5n1p46

\begin{abstract}
Introduction: The current state of our nation's healthcare delivery system, including inefficiencies in healthcare delivery, health disparities, and wasteful costs, accentuates the call for responsive action. Interprofessional education and collaborative practice (IPECP) has emerged as an innovative strategy for addressing many of these inadequacies through the restructuring of healthcare delivery systems and health professions education within the United States. This project was a five-week long course in a clinic setting on interprofessional education (IPE) that supplied basic knowledge of the field of IPECP and offered opportunities to apply knowledge gained in an interprofessional, collaborative manner. The project question was: Will learners exhibit a measurable change in attitudes underpinning a clinic-based culture after exposure to an IPE intervention aimed at guiding and shaping collaborative practice?
\end{abstract}

Methods: This was a systems change project that implemented and assessed a pilot IPECP program. The IPE intervention consisted of a course comprised of two face-to-face sessions and five online modules for 15 pre- and post-licensure learners. Objectives were defined, and mixed methods were used for measurement and assessment, including a pre- and post- test design.

Results: Pre-licensure learners showed high levels of engagement in the learning activities; post-licensure learners failed to meet online engagement objectives. All course learners demonstrated a shift in readiness to engage in IPECP. Quantitative findings were consistent with the qualitative data generated.

Discussion: Findings suggested the beginnings of a shift in attitudes underpinning organizational culture. Findings also revealed barriers to IP learning and practice, including participant attitudes related to professional identity, assumptions about teamwork, and notions about hierarchy.

Key Words: Interprofessional education and collaborative practice, Interprofessional education, Collaborative practice, Intervention research, Culture change

${ }^{*}$ Correspondence: Christa Cerra; Email: CHC221@ pitt.edu; Address: Schools of the Health Sciences Health Policy Institute, University of Pittsburgh, Pittsburgh, United States 
"In Africa there is a concept known as unbutu- the profound sense that we are human only through the humanity of others; that if we accomplish anything in this world, it will in equal measure be due to the work and achievements of others." - Nelson Mandela ${ }^{[1]}$

\section{Introduction}

Research has identified numerous and extensive problems within the United States (US) healthcare delivery system. Healthcare in the US costs more than in any other developed country, without providing better quality. ${ }^{[2]}$ Compromises in patient safety have led to an estimated 1.7 million hospital-associated infections each year, causing approximately 100,000 deaths annually. ${ }^{[3]}$ System inefficiencies are rampant; for example in 2005 , more than $18 \%$ of adults age 65 and over received potentially inappropriate prescription medications. ${ }^{[3]}$ Wasteful costs also burden the healthcare system, such as the $\$ 19$ million spent on medical errors in 2008 , or the $50 \%$ of all annual personal bankruptcy filings in the US that are attributed to medical bills. ${ }^{[3]}$ These concerns led Berwick et al. ${ }^{[4]}$ to suggest the Triple Aim as a generalized approach to fixing the US health care system by calling for three things: 1) improving the patient experience of care, 2) improving the health of populations, and 3) reducing the per capita cost of health care.

The Triple Aim $^{[4]}$ also reinforces the possible importance of interprofessional education and collaborative practice (IPECP) as an approach to assisting with health care redesign and reform. Since the mid-1970s, educators, health professionals, health care researchers, and policy makers have acknowledged that IPECP has the potential to play a key role in improving health care delivery and health outcomes. ${ }^{[5]}$ Research indicates that patients are more apt to receive better quality care when healthcare professionals routinely communicate with one another, work effectively as a team, and have an understanding of the roles and responsibilities of their colleagues from different professions. ${ }^{[6,7]}$ Connecting IPECP with health care redesign has also been reinforced by the World Health Organization (WHO). ${ }^{[8]}$

In 2010, the WHO proposed a definition of interprofessional education (IPE) that has been widely adopted: IPE occurs when "...students from two or more professions learn about, from, and with each other to enable effective collaboration and (to) improve health outcomes." ${ }^{[8]}$ In addition, the United Kingdom's Centre for the Advancement of Interprofessional Education (CAIPE) defines collaborative practice (IPC) as follows: “...interprofessional collaborative practice happens when multiple health-related workers from different professional backgrounds work together with patients, families, care givers and communities to deliver the highest quality of care.'[9]

In 2011 in the US, the Interprofessional Education Collaborative (IPEC) identified 38 core competencies in four do- mains for interprofessional collaborative practice. ${ }^{[10]}$ Pragmatically, these competencies built upon the WHO's ${ }^{[8]}$ definition of collaborative practice and were designed to prepare “...all health professions students for deliberatively working together with the common goal of building a safer and better patient-centered and community/population-oriented US health care system.'[10]

Despite the alignment of large organizational entities and stakeholders enthusiastic about the importance of IPECP, the 40-year history of this area of research has produced scant evidence supporting its outcomes and impact; little is available from which to translate, develop, implement, and evaluate IPECP-related interventions. ${ }^{[11]}$ Clinical sites are challenged with stepping forward into unknown territory to lay the foundation for this advent in patient care. They are summoned to create new approaches to the development of interprofessional learning and collaborative practice, with the ultimate goal of positively impacting the Triple Aim. ${ }^{[4,12]}$

The National Center for Interprofessional Practice and Education (hereafter the National Center) challenges sites to strive toward achieving the Triple Aim by, “...identifying new competencies needed for health professionals, ...test(ing) new organizational and care delivery models, ....and develop(ing), pilot(ing), and implement(ing) curriculum for collaborative care."[13] This project was undertaken to contribute to the evidence base of the field of IPECP by engaging in the above tasks set forth by the National Center.

This paper reports on a pilot systems change project that consisted of designing, implementing, and assessing the impact of an IPE course focusing on providing basic knowledge underpinning the IPEC core competencies, as well as providing opportunities to apply the knowledge gained in an interprofessional, collaborative manner. ${ }^{[14]}$ Within this project, the term "learners" refers to both pre- and postlicensure participants. This reflects the spirit of IPECP, in which both pre- and post-licensure learners are students of interprofessional education, and interprofessional collaborative practice is not limited to the post-licensure population. The pilot took place in a women's health clinic located in a large Midwestern city. The clinic was in the early stages of implementing IPECP.

Staff member roles at the project site included ancillary front-desk staff, medical assistants, registered nurses, behavioral health providers, midwifery and women's health advanced practice nurses, pharmacists, family physicians, OBGYN physicians, a functional nutritionist, a traditional Chinese medicine practitioner, and a reiki practitioner. The site was and remains a teaching clinic for students from various healthcare professional backgrounds (pharmacy, advanced practice nursing and medicine). Several staff members at the clinic hold teaching positions at the clinicaffiliated public University. 
The short-term goal of the course was to lay the foundation for the beginning of a shift in culture within the project site toward a more effective form of IPECP. The long-term goal of the project was to positively impact site-level outcomes, as defined by the Triple Aim. ${ }^{[4]}$ The project question was as follows: Will learners exhibit a measurable change in attitudes underpinning a clinic-based culture after exposure to an IPE intervention aimed at guiding and shaping collaborative practice?

\section{Method}

This was a systems change project that implemented and assessed a pilot IPECP program at a single site. This site was chosen because it was in the early stages of IPECP implementation and identified itself as such. This section describes the intervention and the project assessment design.

\subsection{Project intervention}

To develop the intervention, the site was assessed for strengths and weaknesses to teach, learn, and practice interprofessionally. An earlier version of the InSITE tool, ${ }^{[15]}$ still in the process of being psychometrically validated, was used for site assessment. Use of this tool helped to focus the intervention on areas of weakness. Anonymous comments were informally elicited from post-licensure providers (certified nurse assistants, physicians, advanced practice nurses, and ultrasound technician) and staff at the practice site concerning attitudes toward IPECP.

The intervention was also based on two extensive reviews of the literature, one on IPE and the other on IPC. ${ }^{[14]}$ Both literature reviews were conducted using specific research strategies previously developed and used by experts in the field. ${ }^{[14]}$ The literature reviews yielded a limited amount evidence in the field of IPECP. Thus, in order to develop a relevant intervention, it was important to conduct interviews with experts regarding course structure and content development. The resulting intervention used in this study was developed after consultation with affiliates from the $\mathrm{Na}$ tional Center. ${ }^{[16]}$ The consultations generated the following six concepts and requirements:

(1) Learners should have a theoretical knowledge base in IPECP to assist in effectively developing appropriate skills.

(2) The intervention should supply opportunities for learners to transfer theory into practice.

(3) Learners' experiences should advance behavioral examination of self and team.

(4) The intervention should encompass deliberate, wellstructured learning activities to support collaborative practice.

(5) Learners should be supported in making sense of information by using cluster maps to highlight key themes generated throughout the course. ${ }^{[17]}$
(6) Learning activities should be designed for ease of transferability to other health care settings.

The project intervention consisted of a five-week course in IPE comprised of two face-to-face sessions and five online modules. ${ }^{[18]}$ The course was hybridized in order to accommodate learners' varying schedules, and for ease of transferability across practice settings. Face-to-face sessions took place on weeks 1 and 5 of the course, and online modules took place during weeks 2, 3, and 4 of the course. At the beginning of each face-to-face session, a patient case was presented, followed by interprofessional learning activities (a small group followed by a large group activity). The structure and content of face-to-face sessions during weeks 1 and 5 can be found in Tables 1 and 2, respectively. A description of the small and large group interprofessional learning experiences from each face-to-face session are discussed below.

\subsection{Project population}

The majority of the pre-licensure learners who were scheduled to rotate through the clinic at the time of the course were required to participate in all five weeks of the program. Post-licensure learners were recruited informally through word of mouth and encouraged by the clinic leadership to participate in the training. The professions recruited included pharmacy, reiki, nursing, medicine, and traditional Chinese medicine. Pre-licensure learners had two to four years of health sciences education, and post-licensure learners had a range from four to 20 practice years. Participants had varying degrees of formal exposure to IPE.

\subsection{Small group IPE learning experiences}

During the face-to-face sessions, learners were split into four groups. After presentation of a patient case, each group of learners (pre- and post-licensure) was provided with three questions to discuss. Questions were designed to reflect specific competencies within the IPEC core competency areas of teams and teamwork and roles/responsibilities. Groups were given twenty minutes to move systematically through the questions. One participant from each group transcribed the main talking points from each question.

The week 1 face-to-face session questions were prepared to sequentially guide learners through the process of identifying the following: one's individual role on the team in caring for the patient, the roles and responsibilities of other members of the team in caring for the patient, and team-based actions to support caring for the patient. The competencies and corresponding questions for the first face-to-face session were as follows:

(1) Recognize one's limitations in skills, knowledge, and abilities: What are my roles and responsibilities in caring for this patient ? $^{[10]}$ 
(2) Explain the roles and responsibilities of other care providers and how the team works together to provide care: Based on the previous discussion about roles and responsibilities, what have I learned about the other professional roles of my group members in caring for this patient? ${ }^{[10]}$

(3) Engage other health professionals, appropriate to the specific situation, in shared, patient-centered care: Discuss ways to foster involvement of the whole team in patient-centered care for this patient. ${ }^{[10]}$

Table 1: Week 1 Face-to-Face structure and content

\begin{tabular}{|c|c|c|}
\hline $\begin{array}{l}\text { Length of Time } \\
\text { In Minutes }\end{array}$ & Location & Activity \\
\hline 10 & Large group classroom & $\begin{array}{l}\text { Large group assembled } \\
\text { Questionnaires distributed and completed included: } \\
\text { - } \quad \text { Pre and Post IPE Assessment Questionnaire; and } \\
\text { - } \quad \text { Demographics Questionnaire }\end{array}$ \\
\hline 20 & Large group classroom & Clinic introduced \\
\hline 10 & Large group classroom & $\begin{array}{l}\text { Handouts distributed and reviewed included: } \\
\text { - } \quad \text { Course Information Handout; and } \\
\text { - Week } 1 \text { Face-to-Face Objectives and Agenda } \\
\text { Presentation consisting of: } \\
\text { - } \quad \text { Video viewing (University of Minnesota Academic Health Center } \\
\text { - Learning Commons, 2012): http://youtu.be/IqpT95TKumY; and } \\
\text { Introduction to IPECP }\end{array}$ \\
\hline 5 & Large group classroom & $\begin{array}{l}\text { Patient case presented } \\
\text { Large group broke off into three groups of four and one group of five }\end{array}$ \\
\hline 20 & Small group classroom & Small groups engaged in IPE learning experience \\
\hline 10 & $\begin{array}{l}\text { Restrooms and small group } \\
\text { classrooms }\end{array}$ & Break \\
\hline 40 & Large group classroom & $\begin{array}{l}\text { Small groups reconvened into one large group } \\
\text { Large group engaged in IPE learning activity }\end{array}$ \\
\hline 2.5 & Large group classroom & $\begin{array}{l}\text { Closing remarks consisted of } \\
\text { - A review of IPECP concepts; and } \\
\text { Encouraging participants to experiment with actively applying new } \\
\text { concepts to their experiences in the clinic }\end{array}$ \\
\hline 2.5 & Large group classroom & $\begin{array}{l}\text { Course reminders consisted of: } \\
\text { - Completing the online IPE learning activities and discussion forums } \\
\text { while in the clinic; and } \\
\text { - Contacting the project lead with any questions about working with the } \\
\text { Moodle interface }\end{array}$ \\
\hline
\end{tabular}

Questions for the second face-to-face session during week five were structured to lead the team into developing an actual care plan for the patient case under discussion. The competencies and corresponding questions for the second face-to-face session were as follows:

(1) Recognize one's limitations in skills, knowledge, and abilities: How will each profession on the team be incorporated into the care plan? ${ }^{[10]}$

(2) Explain the roles and responsibilities of other care providers and how the team works together to provide care: How will you coordinate care for this patient with the rest of the team [10] $^{[10}$

(3) Engage other health professionals, appropriate to the specific situation, in shared, patient-centered care: In- corporating items one and two, develop a plan of care for this patient. ${ }^{[10]}$

\subsection{Large group IPE learning experiences}

After the small group activities and a break, the participants re-convened and were given five minutes to quietly review all of the groups' responses. In the first face-to-face session during week one, the content was discussed in the large group in a sequential manner using the following three questions:

(1) What are the common themes in the responses to the questions?

(2) Who will do what for this patient?

(3) What is our final plan of care for the patient? 
At the second face-to-face session during week five, small group responses were also reviewed and discussed at the second face-to-face by guiding the large group through the following series of questions in a sequential manner:

(1) How will each profession on the team be incorporated into the care plan?

(2) How will you coordinate care for this patient with the rest of the team?

(3) How will you develop a plan of care for this patient incorporating items one and two?

Table 2: Week 5 Face-to-Face structure and content

\begin{tabular}{|c|c|c|}
\hline $\begin{array}{l}\text { Length of Time } \\
\text { In Minutes }\end{array}$ & Location & Activity \\
\hline 5 & Large group classroom & $\begin{array}{l}\text { Large group assembled } \\
\text { Week } 5 \text { Face-to-Face Objectives, Outcomes, and Format handout } \\
\text { distributed and reviewed }\end{array}$ \\
\hline 5 & Large group classroom & $\begin{array}{l}\text { Cluster maps reviewed from on-line IP learning activities from Week } 3 \text { and } \\
\text { Week } 4\end{array}$ \\
\hline 5 & Large group classroom & $\begin{array}{l}\text { Patient case presented } \\
\text { Large group broke off into four groups }\end{array}$ \\
\hline 20 & Small group classroom & Small groups engaged in IPE learning experience \\
\hline 5 & $\begin{array}{l}\text { Restrooms and small group } \\
\text { classrooms }\end{array}$ & Break \\
\hline 45 & Large group classroom & $\begin{array}{l}\text { Small groups reconvened into one large group } \\
\text { Large group engaged in IPE learning experience } \\
\text { Participants asked to once again assume same small groups they were in for } \\
\text { the IPE small group exercise }\end{array}$ \\
\hline 7 & Small groups classrooms & $\begin{array}{l}\text { Small groups engaged in an end-of-course exercise by discussing and } \\
\text { identifying the three most important points that they learned in the course }\end{array}$ \\
\hline 8 & Large group classroom & $\begin{array}{l}\text { Small groups reconvened into one large group } \\
\text { Each small group sharped their most important points with the large group }\end{array}$ \\
\hline 5 & Large group classroom & $\begin{array}{l}\text { Course closing remarks delivered consisting of: } \\
\text { - } \quad \text { Brief comments on relationship between IPECP and the Triple Aim; } \\
\text { Video viewing (Institute of Medicine, n.d.): } \\
\text { http://resources.iom.edu/widgets/vsrt/healthcare-waste.html } \\
\text { Acknowledgements and expression of appreciation to clinic } \\
\text { leadership and staff for hosting the course }\end{array}$ \\
\hline 10 & Large group classroom & $\begin{array}{l}\text { Questionnaires distributed and completed included: } \\
\text { - } \quad \text { Pre and Post IPE Assessment Questionnaire } \\
\text { - } \quad \text { On-line Participation Questionnaire; and } \\
\text { - } \quad \text { End-of-Course Feedback Form }\end{array}$ \\
\hline
\end{tabular}

\subsection{Online module interventions}

The online portion of the course was developed using the Moodle electronic interface (version 2.6, Moodle PTY LTD, Perth, Australia). ${ }^{[19]}$ This offers a secure, online learning stage enabling the creation of learning platforms, including weekly lesson plans, discussion forums, and links to videos and outside websites and resources.

There were five online modules. The modules for Weeks 1 and 5 presented materials and content used during the two face-to-face sessions. The modules for weeks 2,3 , and 4 were self-guided and were not followed by a face-to-face session. They consisted of a presentation of IPECP-related background material, a clinic-based IPE learning experience through which to apply the material, and a guided discussion forum.

\subsection{Measures}

Demographic data were collected on all of the participants and on participation rates in both the face-to-face sessions and online discussions. A survey collected the demographic information, and face-to-face participation was tracked using a pre- and post-modified Readiness for Interprofessional Learning Scale (RIPLS). ${ }^{[20]}$ Online participation was tracked using the Moodle interface. Target participation 
rates were set at 70 percent for all of the intervention activities. This was an arbitrary rate but was chosen because it was higher than 50 percent but not so onerous that it would be too difficult to be met.

Cluster maps were created to present key themes found in the qualitative data generated by participants throughout the course. These cluster maps pertained to the two IPEC core competency areas of teamwork and team-based care and roles and responsibilities. To create the cluster maps, key themes were pulled out from artifacts produced from both the online and face-to-face sessions. The online artifacts were the written responses to questions posed in the discussion forums. The face-to-face artifacts were the scribed responses to questions posed by the project lead.

There were two project outcome objectives. The first was related to the IPEC core competencies of teamwork and team-based care: participants will show a statistically significant improvement in readiness to engage interactively with others. ${ }^{[20]}$ The second was related to roles and responsibilities: participants will show a statistically significant improvement in readiness to engage interactively with others. ${ }^{[20]}$ RIPLS, ${ }^{[20]}$ a 5-point, Likert-type, pre- and posttest, ordinal measurement questionnaire, was modified (with permission from authors) to measure for both outcome objectives. The 2006 version of RIPLS was used and not the 1999 version. The objectives which measured participant readiness to engage in interprofessional learning were related to statements about teamwork and collaboration, and a sense of professional identity.

Statements in the RIPLS questionnaire are divided into three principal factors. Factor 1 contains thirteen statements assessing for readiness to engage in interprofessional learning related to teamwork and collaboration. Factor 2 contains five statements assessing for participant readiness to engage in interprofessional learning related to patient-centeredness. Factor 3 contains five statements assessing for readiness to engage in interprofessional learning related to participant sense of professional identity. To reflect the two IPEC core competency domains chosen for the course, the RIPLS questionnaire was modified to include only Factor 1 and 3 questions.

\subsection{Data collection}

The outcome objectives were evaluated using a one group pre- and post-test design. The pre- and post-test design allows for measurement of the impact of the educational experience. During the first ten minutes of the first face-toface session in Week 1 and the last ten minutes of the last face-to-face session in Week 5, learners completed the modified RIPLS. To preserve anonymity and still enable individual tracking of the questionnaires, learners created an alias name that they used pre- and post- exposure to the intervention.

Published by Sciedu Press

\subsection{Data analysis}

A dependent paired $t$-test analysis was performed to ascertain statistical significance between pre- and post-means for the outcome objectives. SPSS (version 21.0, IBM, Chicago, Illinois) was used for the analysis and alpha was set at $p<.05$.

\subsection{Human subjects}

Upon review, the University of Minnesota Institutional Review Board deemed the project to be a quality improvement project that did not meet the threshold for further human subjects review. With respect to participant privacy, the study was considered to be of minimal risk to participants; obtaining learner consent was not required.

\section{Results}

Fifteen learners participated; 10 were pre-licensure health care students and five were licensed providers. Pre-licensure learners consisted of one pharmacy student, two advanced practice nursing doctoral students, and six medical students. Post-licensure learners consisted of one reiki therapist, one traditional Chinese medicine practitioner, one pharmacist, and two physicians.

Eight out of 10 pre-licensure learners had completed a semester-long course on IPE during their first year of schooling. One post-licensure learner had been formally involved with IP activities, trainings, and workshops for over 20 years. The two remaining pre-licensure healthcare students and four remaining post-licensure learners had not previously received any formal training in the field of IPECP. The group represents a convenience sample.

The project lead completed the site assessment using InSITE. ${ }^{[15]}$ This assessment revealed several strengths such as: a high level of readiness to teach, practice, and learn interprofessionally; motivation by some individuals to learn more about the field; and provider efforts to move toward a more collaborative, interprofessional practice. The assessment also revealed barriers concerning readiness to teach, practice, and learn interprofessionally, including a lack of knowledge of the field (see Table 3).

The site assessment also revealed varying staff and provider attitudes towards IPECP. For example, anonymous comments gathered displaying insight into and support for IPECP included the following:

(1) I see some of the areas in which my colleagues are stuck believing that they are practicing teamwork just because there are a wide variety of healthcare disciplines working in the same office space. It will require a little more work to help them understand what teamwork is all about. 
(2) I am energized when watching and facilitating student engagement in IP learning.

(3) The clinic has a long way to go. What we have started is only just the beginning.
(4) I'd like to learn more about new developments in the field. Where do I start?

(5) What does interprofessional education mean?

Table 3: Strength and weaknesses of project site readiness to teach, learn, and practice interprofessionally

\begin{tabular}{|c|c|c|}
\hline $\begin{array}{l}\text { InSITE }{ }^{[10]} \\
\text { Domains }\end{array}$ & $\begin{array}{l}\text { Evidence of Readiness to Engage in IP Education, } \\
\text { Learning, and Practice }\end{array}$ & $\begin{array}{l}\text { Barriers to Engaging in IP Education, } \\
\text { Learning, and Practice }\end{array}$ \\
\hline $\begin{array}{l}\text { Site } \\
\text { Infrastructure }\end{array}$ & $\begin{array}{l}\text { Action \#1: Integrative care conferences (ICC) over the noon } \\
\text { hour to discuss patient cases in an informal, impromptu } \\
\text { manner } \\
\text { Goals of the Action \#1: To provide team members with the } \\
\text { opportunity to: } \\
\text {-discuss techniques for how to work more effectively with } \\
\text { particular patients } \\
\text {-build collegial relationships with one another } \\
\text {-express frustrations }\end{array}$ & $\begin{array}{l}\text { Systematic, planned pathway for IPC } \\
\text { collaboration not yet established, including } \\
\text { distinct goals and objectives for IPC } \\
\text { Steps not yet taken steps to formalize } \\
\text { partnerships with local community resources in } \\
\text { order to work towards common goals of } \\
\text { improving population health }\end{array}$ \\
\hline $\begin{array}{l}\text { Organizational } \\
\text { Structures }\end{array}$ & $\begin{array}{l}\text { Action \#1: Established organizational support for the } \\
\text { development of IPE, as demonstrated by: } \\
\text {-a verbally stated commitment to the systems change project } \\
\text { from the medical director } \\
\text {-request for access to the online portion of the course from the } \\
\text { organizational department chair in order to review course } \\
\text { content and monitor ongoing progress and development } \\
\text { Goals of Action \#1: } \\
\text {-To support a shift in culture } \\
\text {-To acknowledge awareness of potential benefits of improved } \\
\text { IP teamwork }\end{array}$ & $\begin{array}{l}\text { Systematic, planned pathway for } \\
\text { interprofessional education not yet established } \\
\text { Not all staff provided with opportunity to } \\
\text { contribute to leadership decision to formalize } \\
\text { IPE at the practice site }\end{array}$ \\
\hline $\begin{array}{l}\text { Preceptor } \\
\text { Qualities }\end{array}$ & $\begin{array}{l}\text { Action \#1: Select group of site preceptors routinely observed } \\
\text { seeking clinical input from disciplines other than their own } \\
\text { regarding real-time, complex patient cases } \\
\text { Goals of Action \#1: } \\
\text {-To develop the most effective plan of care for complex } \\
\text { patient cases in order to improve health outcomes } \\
\text {-To increase efficiency of healthcare delivery }\end{array}$ & $\begin{array}{l}\text { Preceptors at the site had no formal training in } \\
\text { IPE or IPC }\end{array}$ \\
\hline $\begin{array}{l}\text { Student } \\
\text { Training }\end{array}$ & $\begin{array}{l}\text { Action \#1: Health professional students provided with } \\
\text { opportunities to shadow various healthcare disciplines as part } \\
\text { of their clinical experience while rotating through the site } \\
\text { Action \#2: Joint nurse midwife-medical student orientations } \\
\text { for individuals entering into their obstetrics and gynecology } \\
\text { OBGYN rotations } \\
\text { Goals of Action \#1 \& \#2: } \\
\text {-Increase student understanding of the roles and } \\
\text { responsibilities of professions other than their own }\end{array}$ & $\begin{array}{l}\text { The site was lacking in systematic and/or } \\
\text { planned activities in IPE }\end{array}$ \\
\hline Site Culture & $\begin{array}{l}\text { Action \#1: The project site routinely hosted on-site classes for } \\
\text { patients in the community on women's health and wellbeing } \\
\text { Goals of Action \#1: } \\
\text {-To engage innovative approaches to patient care }\end{array}$ & $\begin{array}{l}\text { General lack of awareness among many site } \\
\text { leaders for cutting edge developments in the field } \\
\text { of IPECP, such as the } 2009 \text { formation of the } \\
\text { IPEC, IPEC's publication of the } \\
\text { interprofessional competencies, and the recent } \\
\text { appointment of the National Center for } \\
\text { Interprofessional Practice and Education }\end{array}$ \\
\hline
\end{tabular}


Anonymous comments gathered from staff displaying barriers to progress included the following:

(1) I've been practicing for years with providers from all kinds of healthcare backgrounds... reiki, acupuncture, nursing... I don't need any formal faculty training in order to teach and practice IEPCP, I am already doing it.

(2) This is a waste of time. It's about academics with their heads in the clouds instead of down on the ground delivering good patient care.

(3) I practice teamwork every day; over half the patients I see on a daily basis are also seen that same day by our PharmD.

(4) I'd like to learn more about it, but I just don't have the time.

(5) Do you have proof that it (IPECP) works?

An analysis of the cluster maps yielded a transformative understanding of the basics of the IPEC-related competencies being measured. Figure 1 cluster maps illustrate learners' application of knowledge during team-based experiences (strategies to address challenges during teamwork, and developing qualities of effective team-based care). Key themes found in these maps included the value of selfreflection and self-confidence to the team experience, adopting an open and curious attitude, and maintaining a willingness to problem-solve with the team. Cluster maps in Figure 2 illustrate learner impressions of the value of understanding other professions' roles and responsibilities (practice improvement, and value of interdisciplinary understanding), including the delivery of more efficient and effective patient care and the strengthening of interdisciplinary, collegiate relationship. Cluster maps in Figure 3 demonstrate learners' awareness of the patient experience of care received (benefits of team based care, and benefits of understanding roles of other professions), including improving patient trust for the team, providing consistent messaging, avoiding repeat work-ups and multiple visits, and developing a more uniform and sensible plan of care.

Participation targets for both domains of the intervention (face-to-face sessions and online modules) were set at $70 \%$. Results were as follows:

(1) $100 \%$ of all enrolled course participants attended both face-to-face sessions.

(2) Pre-licensure learners met the target participation rates for the online discussions and modules; at least $70 \%$ posted at least once in each of the three on-line discussions, and logged onto the website at least twice per week during each week of the course.

(3) Post-licensure learners did not meet the participation rates for either the online discussions or modules (participation began strong but waned).
The first outcome objective (statements about teamwork and collaboration) was not met. For this outcome objective, 1 was the desired response on a five-point Likert-type scale with 1 measuring strongly agree and 5 measuring strongly disagree. The mean pre-test score was 1.59 and the mean post-test score was 1.41. The difference between the means was not statistically significant $(t=-1.62, p=.128)$.

The project's second outcome objective (statements related to a sense of professional identities) was met. For this outcome objective, 5 was the desired response on a five-point Likert-type scale with 1 measuring strongly agree and 5 measuring strongly disagree. The mean pre-test score was 3.91 compared to mean post-test score of 4.15. On average, individuals increased their response by 0.24 on the scale from one to five $(t=2.67, p=.018)$.

\section{Discussion}

This course provided a basic foundation in IPECP for a preand post-licensure population with little to no formal training in the field. The course supplied foundational content and opportunities that sought to transform theory into practice. The online portion of the course served as a vehicle to supply foundational content, enabling learners to fit coursework into their schedules. The face-to-face portion of the course offered opportunities to create a shared patient care plan, to examine attitudes of oneself and others, to actualize the IPEC core competencies, ${ }^{[10]}$ and to reflect on what makes an effective interprofessional learning experience.

Findings from the site assessment revealed that although the site was actively implementing IPECP-related interventions before initiation of the project, attitudes towards engagement were still mixed. Some were in support of the endeavor. Others possessed a limited understanding of the field, and/or displayed attitudinal limitations, including assumptions about professional roles and their connections to hierarchy, territorialism, and power, all of which influenced the development, implementation, and assessment of the course.

For example, although the intervention called for mixing pre- and post-licensure learners in the on-line discussion forums, leadership at the project site preferred that pre- and post-licensure learners be placed in separate discussion forums and be invisible to one another. These choices may have influenced participation rates between pre- and postlicensure learners, calling attention to the potential importance of combining learner populations. Such attitudinal barriers underscore some of the complexities of implementation and the importance of maintaining ongoing program assessment in order to respond effectively and appropriately to ever changing interpersonal and environmental dynamics. ${ }^{[17]}$

Despite these findings, project results suggested the initia- 
tion of a change in attitudes toward more effective forms of interprofessional learning and team functioning. These attitudinal changes might suggest the movement toward a culture shift of greater interprofessionalism. The learners developed a shared understanding of each other that was different from where each of them started individually. Qualitative findings displayed in cluster maps regarding effective team functioning (see Figure 1) illustrated learners synthesizing and applying foundational concepts related to teamwork and collaboration. The second outcome objective pertaining to roles and responsibilities demonstrated a statistically significant improvement in learner readiness to engage others. Cluster map analysis revealed that learners were able to step beyond their own professional perimeters by commenting on the value of the roles and responsibilities of professions other than their own (see Figure 2). They were also able to articulate how understanding the roles and responsibilities of their team members can improve practice (see Figure 2).

Learners were also able to identify benefits to the patient when team members understand each other's roles. Further, learners linked ability to deliver higher quality patient care to factors such as team members maintaining a more open and flexible perspective, sharing patient information with one another, and increasing respect and appreciation for each other's expertise (see Figure 3).

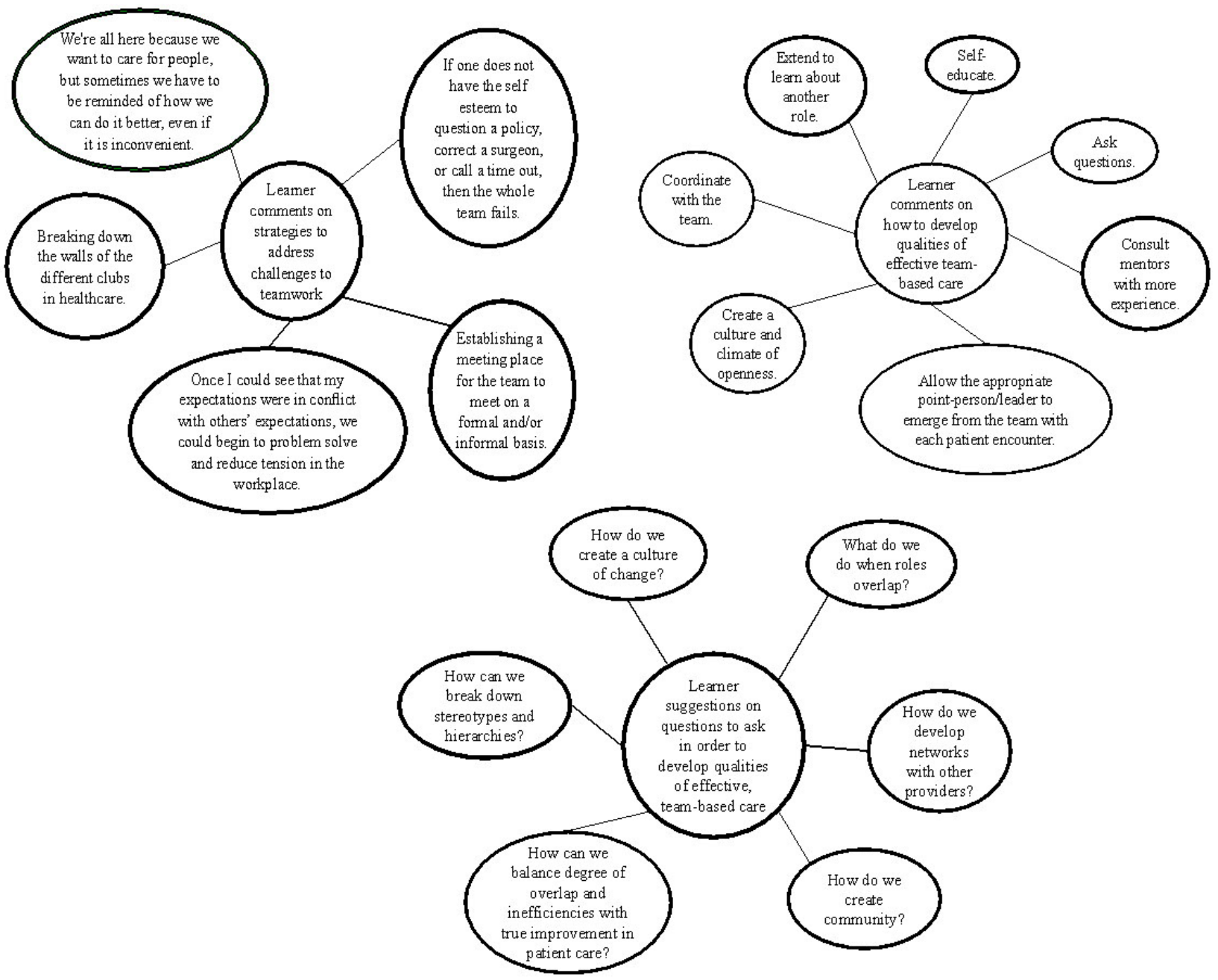

Figure 1: Application of knowledge when examining effective team functioning

\subsection{Limitations}

There were two major limitations to the project. First, project assessment design cannot claim causality; there is no way of knowing if the effects were directly related to the intervention or if some other factors influenced the post- test scores. Second, the RIPLS tool used in this project was designed for use with post-graduate, working professionals only. ${ }^{[20]}$ An earlier version of RIPLS was designed and validated for use with pre-licensure learners. ${ }^{[21]}$ Since a portion of the learners enrolled in the course were healthcare professional students not yet working in a postgraduate, pro- 
fessional role, the RIPLS questionnaire chosen was used in a manner not originally intended. Finally, as a pilot project, a convenience sample was used, the sample size was small, and the project was limited to a single specialty site all of which presents limitations for generalizability of the results.

\subsection{Recommendations for modifying this IPECP in- tervention}

A number of recommendations may aid future course development. First, when implementing IPE interventions, it may be helpful to identify site champions and enroll them in ongoing, formal training in the field of IPECP. After receiving formal training, champions can be used to host future courses. Another recommendation is for course designers to gather ongoing feedback from course participants to aid in making necessary modifications and design improvements for future course content and IPE learning activities.

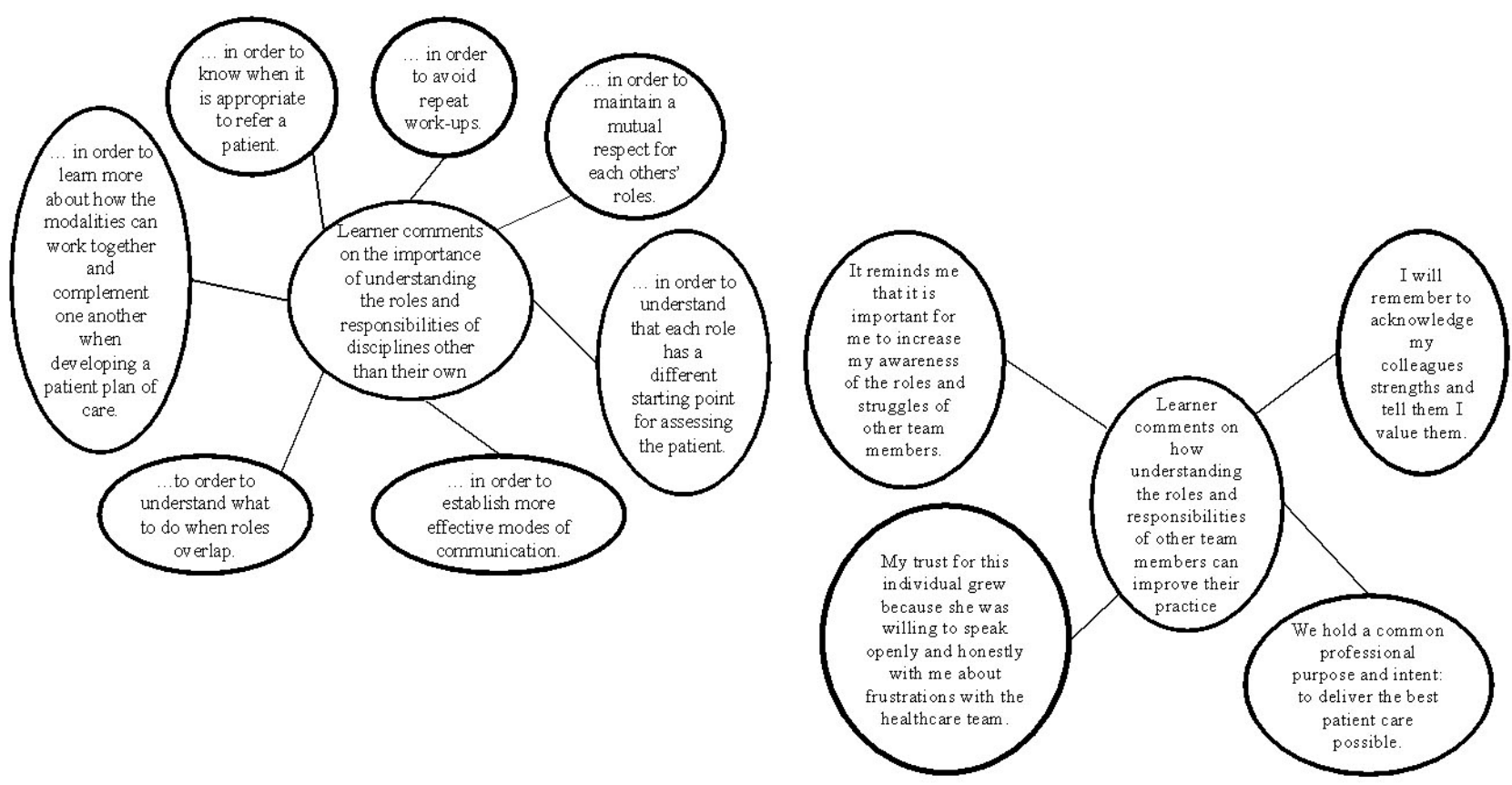

Figure 2: Learner impressions of the value of understanding other professions' roles and responsibilities

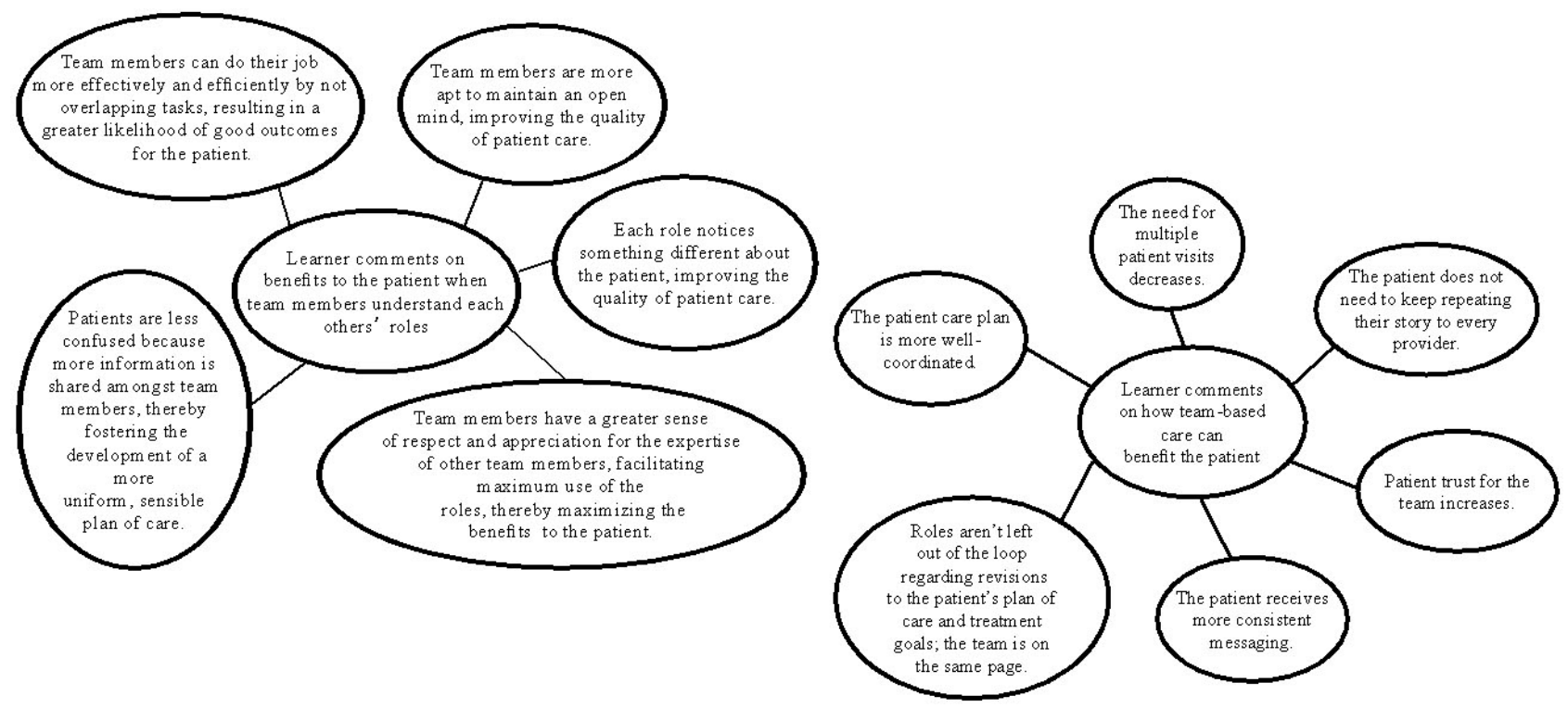

Figure 3: Learner integration and application of the IPEC core competencies of teamwork and team-based care and roles and responsibilities to the patient experiences

Published by Sciedu Press 
Content and IPE learning exercises in the face-to-face sessions and online forums could be easily modified to accommodate sequential courses. For example, practice sites could host two courses on a rotating basis. One course could be focused on the IPEC core competencies of teamwork and team-based care and roles and responsibilities, while the other course could be focused on the two remaining IPEC core competency areas of values/ethics, and interprofessional communication. ${ }^{[10]}$ All current and incoming staff and post-licensure providers at a site could rotate through both courses, along with pre-licensure learners from the various professions as they complete their student rotations.

As IPE champions mature and the clinic acclimates to shifts in culture, it might be possible to change the small group face-to-face sessions from case-based exercises to actual patient care experiences. The face-to-face large group sessions and the online discussion forums could then be used to discuss shared patient care experiences.

\section{Conclusions}

This systems change project consisted of designing, implementing and assessing a course on interprofessional education that supplied basic knowledge on the field with opportunities to apply knowledge in a collaborative manner. The short-term goal of the project was to lay the foundation for the beginnings of a shift in culture within the project site to- ward a more effective form of interprofessional learning and team functioning, with the long-term goal of advancing the Triple Aim. ${ }^{[4]}$

The qualitative and quantitative evidence suggested a change in understanding by the participants that can be used as a building block to initiating a change in culture toward greater receptivity to IPECP. Findings also revealed challenges in working with attitudinal limitations of the participants. To date, such difficulties are likely to be found in any healthcare practice and/or academic setting.

As cultures continue to evolve, meeting these challenges will be an important part of the transformational journey into interprofessional, team-based, patient-engaged care.

\section{Acknowledgements}

In addition to the anonymous reviewers of this manuscript, the authors would also like to thank the following individuals who contributed to different facets of this project: Greg Beilman, Barbara Brandt, Frank Cerra, Kathie Cerra, Leslie Hall, Karla Hemesath, Sue Koska, Jane Miller, Amy Pittenger, Michael Pipestone, Carrie Terrell, Carolyn Torkelson, and Jill Thistlethwaite.

\section{Conflicts of Interest Disclosure}

The author declares that there is no conflict of interest statement.

\section{References}

[1] Stengel S. Mandela's way: Lessons on life, love and courage. New York, New York: Random House Inc, 2009.

[2] Shortell S. Increasing value: a research agenda for addressing the managerial and organizational challenges facing health care delivery in the United States. Med Care Res Rev. 2004; 61: 12s30s. PMID:15375281 http://dx.doi.org/10.1177/1077558 704266768

[3] Agency for Healthcare Research and Quality. National healthcare quality report 2011. 2012. Berwick D, Nolan T, Whittington J. The triple aim: Care, health, and cost. Health Affairs. 2008; 27: 759 769. PMID:18474969 http://dx.doi.org/10.1377/hlthaff .27 .3 .759

[4] Berwick D, Nolan T, Whittington J. The triple aim: Care, health, and cost. Health Affairs. 2008; 27: 759-769. PMID:18474969 http: //dx.doi.org/10.1377/hlthaff .27.3.759

[5] Reeves S, Perrier L, Goldman J, Freeth D, Zwarenstein M. Interprofessional education: Effects on professional practice and healthcare outcomes. Cochrane Database of Systematic Reviews, 2013. PMID:23543515 http://dx.doi.org/10.1002/1465185 8. CD002213.pub3

[6] Barnsteiner J, Disch J, Hall L, Mayer D, Moore S. Promoting interprofessional education. Nursing Outlook. 2007; 55: 144-150. PMID: 17524802

[7] Institute of Medicine. Health professions Education: A bridge to quality. Washington, D.C.: The National Academies Press, 2003.

[8] World Health Organization. Framework for action on interprofessional education and collaborative practice. 2010. Available from: http://whqlibdoc.who.int/hq/2010/WHO_HRH_HPN_ 10.3_eng.pdf?ua=1

[9] Barr H, Waterton S. Interprofessional education in health and social care in the United Kingdom: Report of a CAIPE survey. Centre for the Advancement of Interprofessional Education in Primary Health and Community Care, 1996.

[10] Interprofessional Education Collaborative. Team-based competencies: Building a shared foundation for education and clinical practice. Washington, D.C.: Interprofessional Education Collaborative, 2011.

[11] Brandt BF, Lutfiyya MN, King JA, Chioreso C. A scoping review of interprofessional collaborative practice and education using the lens of the Triple Aim. Journal of Interprofessional Care. 2014; Early Online: $1-7$

[12] Brandt B. Update on the US national center for interprofessional practice and education. Journal of Interprofessional Care. 2014; 28:5-7. PMID:24224866 http://dx.doi.org/10.3109/13561 820.2013 .852365

[13] National Center for Interprofessional Practice and Education, Nexus Innovation Incubator, Testing New Models of Care and Learning. Available from: https://nexusipe.org/innovations-incubator.

[14] Cerra C. Implementing interprofessional education in a clinical practice setting with a mixed student-provider population. A DNP Scholarly Paper Submitted to the Faculty of Nursing of The University of Minnesota in Partial Fulfillment of the Requirements for the Degree of Doctor of Nursing Practice. May 2014.

[15] Regents of the University of Minnesota. InSITE (developed by Sick, B.). 2013. Minneapolis, Minnesota: Regents of the University of Minnesota. 
[16] Brandt B. Update on the US national center for interprofessional practice and education. Journal of Interprofessional Care. 2014 28: 5-7. PMID:24224866 http://dx.doi.org/10.3109/13561 820.2013 .852365

[17] King J, Stevahn L. Interactive evaluation practice: Mastering the interpersonal dynamics of program evaluation. Los Angeles, CA SAGE Publications, 2013

[18] Interprofessional Education in the Clinic Setting. Available from: ht tps://ay14.moodle. umn.edu/course/view.php?id=4466

[19] Moodle.org. About moodle. 2014. Available from: http://docs .moodle.org/27/en/About
[20] Reid R, Bruce D, Allstaff K, McLeron D. Validating the readiness for interprofessional learning scale (RIPLS) in the postgraduate context: Are health care professionals ready for IPL? Medical Education. 2006; 4: 415-422. PMID:16635120 http://dx.doi.org/1 $0.1111 / j .1365-2929.2006 .02442 . x$

[21] Parsell G, Bligh J. The development of a questionnaire to assess the readiness of health care students for interprofessional learning (RIPLS). Medical Education. 1999; 33: 95-100. PMID:10211258 http://dx.doi.org/10.1046/j.1365-2923.1999.00298.x 